\title{
Maximal Controllability of Input Constrained Unstable Systems by the Addition of Implicit Constraints *
}

\author{
Adrian Medioli $^{\mathrm{a}}$, Maria Seron ${ }^{\mathrm{a}}$, Richard Middleton ${ }^{\mathrm{b}}$ \\ ${ }^{a}$ The ARC Centre for Complex Dynamic Systems \& Control, The University of Newcastle, Australia \\ ${ }^{\mathrm{b}}$ The Hamilton Institute, NUI Maynooth, Co Kildare, Ireland
}

\begin{abstract}
The null controllable set of a system is the largest set of states that can be controlled to the origin. Control systems that have a region of attraction equal to the null controllable set are said to be maximally controllable closed-loop systems. In the case of open-loop unstable plants with amplitude constrained control it is well known that the null controllable set does not cover the entire state-space. Further the combination of input constraints and unstable system dynamics results in a set of state constraints which we call implicit constraints. It is shown that the simple inclusion of implicit constraints in a controller formulation results in a controller that achieves maximal controllability for a class of open-loop unstable systems.
\end{abstract}

Key words: Maximal Controllability; Unstable Systems; Input constraints; MPC

\section{Introduction}

The set of all states for which an admissible control sequence exists to steer a current state to the origin in possibly infinite time is known as the null controllable set of a system. Many authors have characterised null controllable sets and formulated controllers which produce closed-loop systems that are asymptotically stable over these sets [14], [13], [7], [4], [12]. We will refer to such closed-loop systems as maximally controllable systems. The work of previous authors has resulted in controllers that may be grouped into several categories. For example, [14] and [12] propose strategies involving the addition of a non-linear anti-windup controller. While [13] and [7] show maximal controllability via the selection of a specific state feedback gain which produces a closedloop system that can arbitrarily closely approximate the null controllable set. In [4] a nonlinear controller is formulated by starting with a set of state feedback gains which are dynamically modified as the null controllable boundary is approached. None of these techniques result in a piecewise linear controller and some like [13] and [7] restrict the state feedback gains to specific val-

\footnotetext{
* The authors acknowledge the support of Matrikon AsiaPacific Pty Ltd, and the Australian Research Council.

Email addresses: adrian.medioli@newcastle.edu.au

(Adrian Medioli), maria.seron@newcastle.edu.au (Maria Seron), Richard.Middleton@nuim.ie (Richard Middleton).
}

ues. In contrast our proposed approach describes a solution that uses a piecewise linear controller structure which under mild assumptions allows the selection of a range of state feedback gains. The desirability of this controller structure stems from a popular advanced optimisation based control strategy known as Model Predictive Control (MPC) (e.g., [2], [9]). More specifically, the explicit solution to MPC is a piecewise linear controller [6]. Hence, both the motivation for the approach and the potential link to MPC are identified throughout the subsequent sections. The work of Blanchini [1], and references therein, most closely resembles the approach in this paper. Their method produces a piecewise linear controller that renders some initial, compact and convex, subset of the region of attraction positively invariant. The controller also guarantees convergence by making this initial set $\lambda$-contractive. However, to approximate a null controllable set, the technique gives a controller that only affects the unstable modes. Hence, our proposed approach has the main advantage of allowing a larger set of controller gains, able to affect both stable and unstable modes, of which theirs is a special case.

The application of MPC with guaranteed stability produces a region of attraction that is a subset of the null controllable set. In general the size of the region of attraction is dependent on the length of the horizon used in the MPC formulation. Therefore, to make the region of attraction of a system equal to the null controllable 
set may involve applying MPC over an infinite horizon. This solution for obtaining the largest possible region of attraction, in the presence of constraints, is intractable. We note that for open-loop asymptotically stable systems an appropriate selection of the MPC parameters means that the region of attraction is the entire statespace and therefore the presented work is not relevant to these types of systems.

In this paper we introduce the concept of implicit constraints. Implicit constraints result from the combination of explicit or applied constraints (such as actuator saturation constraints) and system dynamics (such as plant open-loop instabilities). Implicit constraints are therefore a concept that is closely associated with the null controllable set. A motivating example of a second order unstable system reveals that a maximally controllable closed-loop system results from making implicit constraints "explicit" in the MPC formulation. Further, the resulting closed-loop system is maximally controllable independent of the horizon length used. We then consider a more general class of input constrained unstable systems with any state dimension and one unstable mode. We show that applying a control strategy which enforces implicit constraints results in closed-loop systems with a piecewise affine controller. Under mild assumptions, the properties of the controller in each control region is such that the resulting closed-loop systems achieve a controllable set that is an arbitrarily close approximation to the null controllable set.

The remainder of the paper is divided into eight sections. Preliminaries are covered by three sections beginning with Section 1.1 which provides a motivating example for the application of implicit constraints. Section 2 details the definition of the systems and their null controllable sets, and the statement of a control strategy which enforces implicit constraints is presented in Section 3. The analysis requires the definition of some constructs found in Section 4. Section 5 describes the properties of the various analysis regions and Section 6 combines all of the elements to prove maximal controllability of the closed-loop systems. Finally, an example provides a practical illustration of the presented theory, Section 8 discusses extensions to the technique and some conclusions complete the paper.

\subsection{Implicit Constraints: A Motivating Example}

To motivate the contents of this paper we consider an electromagnetic ball suspension apparatus. This system uses a current $I$ to energise an electromagnet in order to suspend a ferromagnetic ball of mass $m_{b}$ at a desired height $y=y^{*}$. A linearised model of such a system (see [8], Example 12.8) where the ball displacement $y$ and the ball velocity $v$ are the system states is given as

$$
\left[\begin{array}{l}
\dot{y} \\
\dot{v}
\end{array}\right]=\left[\begin{array}{cc}
0 & 1 \\
0.385 & -1 \times 10^{-5}
\end{array}\right]\left[\begin{array}{l}
y \\
v
\end{array}\right]+\left[\begin{array}{c}
0 \\
-0.057
\end{array}\right] I .
$$

If the current available to energise the electromagnet is limited, for example $I \in[-10,10]$, we implicitly limit the minimum height at which the ball can be suspended. More specifically by performing a similarity transformation such that the system's unstable state $x_{1}$ is separate (i.e., $\dot{x}_{1}=a_{1} x_{1}+b_{1} I$ ) we can determine that the input constraints produce the limit $x_{1} \in\left[10 b_{1} / a_{1},-10 b_{1} / a_{1}\right]$. For this example, transforming the limits on $x_{1}$ back to the original state-space results in the limits $x_{1}=$ $y+0.051 v \in[-1.5,1.5]$. This observation highlights that external constraints in combination with the dynamics of a system introduce what we term implicit constraints. Figure 1 shows a portion of the state-space of the ball for

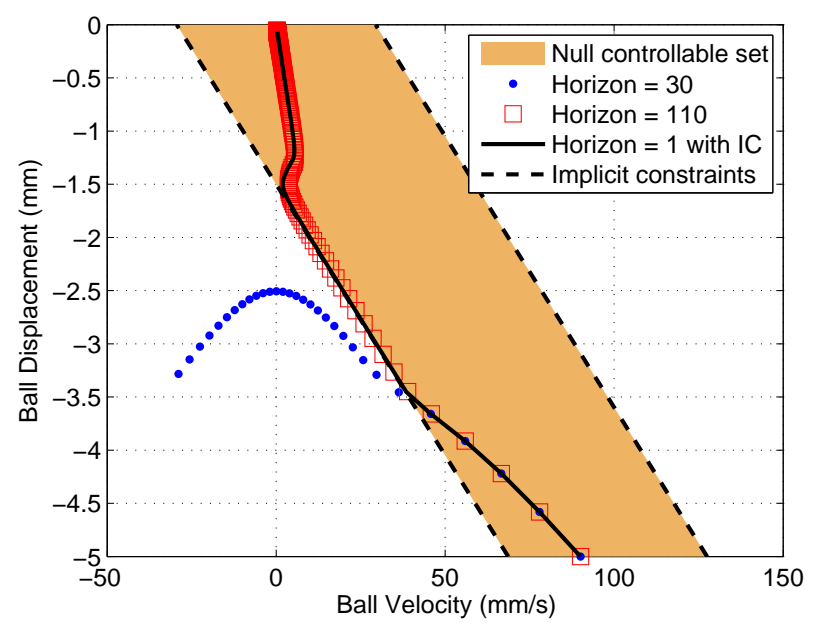

Fig. 1. Magnetic suspension system trajectories.

the linearised model (1). The shaded area of the graph is a portion of the null controllable set and the dashed lines are the implicit constraints resulting from the minimum and maximum available current. The curves plotted in Fig. 1 correspond to three state-space trajectories resulting from different formulations of MPC applied to an initial state $[90-5]^{T}$. The objective is to bring the state to zero.

Each of the three MPC formulations is implemented with a state weighting $Q=1 \times 10^{6} I$, input weighting $R=1$, terminal state weighting $P_{N}$ being the solution to the discrete-time algebraic Riccati equation and without terminal state constraints. Further the MPC problem is formulated with a discrete-time version of the system model with a sampling period of $0.001 \mathrm{~s}$.

Formulating MPC with input constraints and a horizon of length 30 produces the trajectory marked by dots. We 
see that this trajectory exits the null controllable set defined by the available control authority and as such the ball displacement diverges. Formulating MPC with input constraints and a horizon of length 110 produces the trajectory marked by squares. We see that the trajectory does not exit the null controllable set and asymptotically converges to the origin. Comparing these two MPC formulations highlights the fact that the length of the horizon can be selected for any given initial state so that an asymptotically stable trajectory is produced. However, if the horizon is not long enough the system behaves with a degree of "myopia" in the sense that it can not "see" that the optimal trajectory selected will move the state outside the controllable region. Therefore, given the maximum available control action, it becomes impossible to prevent the ball from falling.

From this observation a reasonable conjecture is that if the system were to see the boundary of the null controllable set as a constraint, then the system could avoid exiting this region. This is confirmed for this example by formulating MPC with input constraints and adding "explicitly" the implicit constraint that describes the boundary of the null controllable set, which results in the trajectory shown in Fig. 1 as a solid line. The simulation clearly shows that the introduction of the implicit constraint to the MPC formulation ensures that the trajectory remains within the null controllable set. Further, we may anticipate that stability is independent of the selected horizon length since the solid line trajectory shown in Fig. 1 is for a horizon of one ${ }^{1}$.

The remainder of the article presents a controller which enforces implicit constraints to show situations where the intuition gained from this example is valid.

\section{Problem Formulation}

This section introduces a class of $n^{\text {th }}$ order input constrained systems with one unstable mode and input constraints. We will show in the following sections that, by applying the continuous-time controller described in Section 3 and introducing implicit constraints, the resulting closed-loop system is maximally controllable.

We consider a class of open-loop unstable, linear, time invariant, $n^{\text {th }}$ order systems with the following statespace representation,

$$
\dot{x}=A x+B u
$$

\footnotetext{
1 If a system has a null controllable set which can be easily characterised by a set of implicit constraints, it is possible that adding these implicit constraints to the controller formulation results in a maximally controllable system. At the very least, adding the null controllable set as an explicit constraint cannot decrease the region of attraction.
}

where $x$ is the state vector in $\mathbb{R}^{n}, u$ is the input in $\mathbb{R}$ and $(A, B)$ is controllable. We assume that the matrix $A$ is diagonalisable and that its eigenvalues $\left(\lambda_{1}, \lambda_{2}, \ldots\right.$, $\left.\lambda_{n}\right)$ are real and satisfy $\lambda_{1}>0$ and $\lambda_{i}<0$ for $i=$ $2, \ldots, n$. Under these assumptions, there is no loss of generality in assuming that the block matrices in (2) have the following form,

$$
A=\left[\begin{array}{cc}
a_{u 1} & 0 \\
0 & A_{s}
\end{array}\right], \quad B=\left[\begin{array}{c}
b_{u 1} \\
B_{s}
\end{array}\right]
$$

where $a_{u 1}=\lambda_{1}$ is the only unstable eigenvalue and $A_{s}=$ $\operatorname{diag}\left(a_{s 1}, a_{s 2}, \ldots, a_{s q}\right)$ is a diagonal matrix consisting of $q=n-1$ stable eigenvalues $a_{s i}=\lambda_{i+1}$ for $i=1, \ldots, q$. Accordingly, the state is partitioned as $x=\left[\begin{array}{ll}x_{u 1} & x_{s}^{T}\end{array}\right]^{T}$, $x_{u 1} \in \mathbb{R}, x_{s} \in \mathbb{R}^{n-1}$, where $x_{u 1}$ is the unstable state and $x_{s} \triangleq\left[\begin{array}{lll}x_{s 1} & \ldots & x_{s q}\end{array}\right]^{T}$ is the vector of stable states.

Further we will assume that the input magnitude is constrained, that is $\underline{u} \leq u \leq \bar{u}$ with $\bar{u}>0, \underline{u}<0$ and without loss of generality that $b_{u 1}>0$ and $^{2} B_{s}>0$.

\subsection{Null Controllable Set}

For a system with a single unstable pole the bounds in the direction of the unstable state are determined by the values of the unstable state that can be cancelled in finite time by the minimum and maximum available control magnitudes. Thus, the null controllable region for (2)-(3) denoted by $\mathcal{R}_{\max }$ has the following form [13]:

$$
\mathcal{R}_{\text {max }}=\left\{x \in \mathbb{R}^{n}:-\frac{b_{u 1}}{a_{u 1}} \bar{u}<x_{u 1}<-\frac{b_{u 1}}{a_{u 1}} \underline{u}\right\} .
$$

\subsection{Null Controllable Set Approximation}

Since the null controllable set is open on the unstable state boundaries we will consider for our analysis a closed approximation ${ }^{3}$ to this region, as defined below. Definition 1 (Region $\mathcal{R}_{\max -\varepsilon}$ ) The region $\mathcal{R}_{\max -\varepsilon}$ is
defined as

$$
\mathcal{R}_{\max -\varepsilon} \triangleq\left\{x \in \mathbb{R}^{n}: \underline{x}_{u 1} \leq x_{u 1} \leq \bar{x}_{u 1}\right\}
$$

where

and

$$
\begin{aligned}
& \underline{x}_{u 1} \triangleq-b_{u 1} \bar{u} / a_{u 1}+\varepsilon_{u 1}, \\
& \bar{x}_{u 1} \triangleq-b_{u 1} \underline{u} / a_{u 1}-\varepsilon_{u 1},
\end{aligned}
$$

$$
0<\varepsilon_{u 1}<\min \left\{-b_{u 1} \bar{u} / a_{u 1},-b_{u 1} \underline{u} / a_{u 1}\right\} .
$$

\footnotetext{
2 In the remainder of the article, inequalities applied to vectors are to be considered element wise.

$3 \mathcal{R}_{\max -\varepsilon}$, as per Definition 1 , can be made arbitrarily close to the null controllable set through the selection of $\varepsilon_{u 1}$.
} 


\section{Control}

This section specifies a piecewise affine control law which incorporates the application of implicit constraints. The selected controller is then analysed to detail its behaviour throughout the null controllable region.

$$
u= \begin{cases}\bar{u} & \text { if } x_{u 1}<\underline{x}_{u 1} \\ \operatorname{sat}(-K x) & \text { if } \underline{x}_{u 1} \leq x_{u 1} \leq \bar{x}_{u 1} \\ \underline{u} & \text { if } x_{u 1}>\bar{x}_{u 1}\end{cases}
$$

where $K$ is a state feedback gain and $\operatorname{sat}(v)=\underline{u}$ if $v<\underline{u}$, $v$ if $\underline{u} \leq v \leq \bar{u}$ and $\bar{u}$ if $v>\bar{u}$.

The control law (9) implies that for $\underline{x}_{u 1} \leq x_{u 1} \leq \bar{x}_{u 1}$ the state-space is partitioned into three control regions ${ }^{4}$ (as shown in Fig. 2 for the case $x_{s} \in \mathbb{R}$ ): a linear control region $\underline{u} \leq-K x \leq \bar{u}$, where the linear control $u=-K x$ is applied; and two input constrained regions $-K x<\underline{u}$ and $-K x>\bar{u}$, corresponding to $u=\underline{u}$ and $u=\bar{u}$, respectively. In addition for $x_{u 1}>\bar{x}_{u 1}$ or $x_{u 1}<\underline{x}_{u 1}$ the resulting input is such that $x_{u 1}$ moves back toward the boundary at the maximum rate given the available input range, thus effectively imposing the "implicit constraint" of keeping the system inside an arbitrarily close approximation of the controllable region.

\subsection{Controller Properties}

For some portions of the hyperplanes $x_{u 1}=\bar{x}_{u 1}$ and $x_{u 1}=\underline{x}_{u 1}$ the right hand sides of the differential equations describing the closed-loop system (2)-(3), (9) are discontinuous. For these kinds of differential equations, existence and uniqueness of solutions on the discontinuous portions may be considered using differential inclusions theory as proposed by Filippov [5]. In general discontinuities occur on a switching surface $s(x)=0$ and existence and uniqueness of solutions on this surface in the sense of Filippov can be guaranteed if all points $x^{*}$ in the surface satisfy the following conditions [11].

$$
\lambda_{+}\left(x^{*}\right)<0 \text { or } \lambda_{-}\left(x^{*}\right)>0,
$$

where

$$
\begin{aligned}
\lambda_{+}\left(x^{*}\right) & =\lim _{x \rightarrow x^{*}} \lambda_{+}(x), \lambda_{-}\left(x^{*}\right)=\lim _{x \rightarrow x^{*}} \lambda_{-}(x), \\
x^{*} & \in\left\{x \in \mathbb{R}^{n}: s(x)=0\right\},
\end{aligned}
$$

and

$$
\begin{aligned}
& \lambda_{+}(x)=\frac{d s}{d t}=\frac{\partial s}{\partial x} \dot{x} \text { for } s(x) \in S^{+} \triangleq\left\{x \in \mathbb{R}^{n}: s(x)>0\right\} \\
& \lambda_{-}(x)=\frac{d s}{d t}=\frac{\partial s}{\partial x} \dot{x} \text { for } s(x) \in S^{-} \triangleq\left\{x \in \mathbb{R}^{n}: s(x)<0\right\}
\end{aligned}
$$

\footnotetext{
4 Note that, using this approach the resulting continuoustime closed-loop system is a piecewise affine system similar to a one step horizon MPC formulation of the problem.
}

We consider the case $x_{u 1}=\bar{x}_{u 1}$, the switching surface is given by $s(x)=x_{u 1}-\bar{x}_{u 1}=0$ and $s(x)>0 \Longleftrightarrow$ $x_{u 1}>\bar{x}_{u 1}, s(x)<0 \Longleftrightarrow x_{u 1}<\bar{x}_{u 1}$. By using the system equations (2)-(3) and the control law (9), $\lambda_{+}(x)$ and $\lambda_{-}(x)$ become,

$$
\begin{aligned}
& \lambda_{+}(x)=a_{u 1} x_{u 1}+b_{u 1} \underline{u} \text { for } x_{u 1}>\bar{x}_{u 1}, \\
& \lambda_{-}(x)=a_{u 1} x_{u 1}+b_{u 1} \operatorname{sat}(-K x) \text { for } x_{u 1}<\bar{x}_{u 1} .
\end{aligned}
$$

First we consider $\lambda_{+}\left(x^{*}\right)$. From (11) and $s(x)=x_{u 1}-$ $\bar{x}_{u 1}$ we have $x_{u 1}^{*}=\bar{x}_{u 1}$, hence, from (12), by using (7) results in $\lambda_{+}\left(x^{*}\right)=-\varepsilon_{u 1} a_{u 1}$. Since $a_{u 1}>0, \varepsilon_{u 1}>0$, we have that in $S^{+}$(that is, $x_{u 1}>\bar{x}_{u 1}$ ),

$$
\lambda_{+}\left(x^{*}\right)=-\varepsilon_{u 1} a_{u 1}<0 .
$$

Hence the first condition in (10) is always true, guaranteeing existence and uniqueness of the solution of (2)(3), (9) in the sense of Filippov, on the switching surface.

Let us now consider $\lambda_{-}\left(x^{*}\right)$. Using (13) we then have, that in $S^{-}$(that is, $\left.x_{u 1}<\bar{x}_{u 1}\right), \lambda_{-}\left(x^{*}\right)=$ $a_{u 1} \bar{x}_{u 1}+b_{u 1} \operatorname{sat}\left(-K x^{*}\right)$. We can consider three different cases that define portions of the switching surface for which the trajectories on both sides present different behaviour. These cases are:

$\lambda_{-}\left(x^{*}\right)= \begin{cases}\sigma+b_{u 1} \operatorname{sat}\left(-K x^{*}\right)>0 & \text { if } K_{s} x_{s}^{*}<\alpha_{s} \\ \sigma+b_{u 1} \operatorname{sat}\left(-K x^{*}\right) \leq 0 & \text { if } \alpha_{s} \leq K_{s} x_{s}^{*}<\alpha_{c} \\ -\varepsilon_{u 1} a_{u 1}<0 & \text { if } K_{s} x_{s}^{*} \geq \alpha_{c}\end{cases}$

where $\sigma=a_{u 1} \bar{x}_{u 1}, K=\left[\begin{array}{ll}k_{u 1} & K_{s}\end{array}\right], x^{*}=\left[\bar{x}_{u 1} x_{s}^{* T}\right]^{T}$ and $\alpha_{c}=\underline{u}\left(b_{u 1} k_{u 1}-a_{u 1}\right) / a_{u 1}+\varepsilon_{u 1} k_{u 1}, \alpha_{s}=\underline{u}\left(b_{u 1} k_{u 1}-\right.$ $\left.a_{u 1}\right) / a_{u 1}+\varepsilon_{u 1}\left(b_{u 1} k_{u 1}-a_{u 1}\right) / b_{u 1}$. Conditions (14) and (15) imply that for the portion $K_{s} x_{s}^{*}<\alpha_{s}$ the trajectories on both sides of the switching surface $x_{u 1}=\bar{x}_{u 1}$, point toward it. For the portion $K_{s} x_{s}^{*} \geq \alpha_{s}$ the trajectories in $S^{+}$point toward the switching surface and those in $S^{-}$away from it. In addition, for $\alpha_{s} \leq K_{s} x_{s}^{*}<\alpha_{c}$, the right hand side of (2)-(3), (9) is discontinuous on the switching surface whereas for $K_{s} x_{s}^{*} \geq \alpha_{c}$ it is continuous. (Note that $K_{s} x_{s}=\alpha_{c}$ represents the intersection between the switching surface and the hyperplane $-K x=\underline{u}$ where the controller saturates to $\underline{u}$.) Hence, for $K_{s} x_{s}^{*}<\alpha_{s}$, the trajectories slide along the switching surface $x_{u 1}=\bar{x}_{u 1}$ with an equivalent control $\bar{u}_{e q}$ [3], defined as the control that maintains the condition $\dot{s}(x)=\dot{x}_{u 1}=0$, that is, $\bar{u}_{e q}=a_{u 1} \varepsilon_{u 1} / b_{u 1}+\underline{u}$.

For $\alpha_{s} \leq K_{s} x_{s}^{*}<\alpha_{c}$, it may be shown that the trajectory defined in the sense of Filippov has only $x^{*}$ in common with the switching surface and goes from $S^{+}$to $S^{-}$ through $x^{*}$ [11]. For $K_{s} x_{s}^{*} \geq \alpha_{c}$ the differential equations $(2)-(3),(9)$ have a unique solution in the standard sense and the resulting trajectories cross the switching surface from $S^{+}$to $S^{-}$. Thus, closed-loop system trajectories starting on the hyperplane $x_{u 1}=\bar{x}_{u 1}$ either slide 
on the hyperplane or move towards $S^{-}=\left\{x \in \mathbb{R}^{n}\right.$ : $\left.x_{u 1} \leq \bar{x}_{u 1}\right\}$.

A similar set of arguments can be made for the case of $x_{u 1}=\underline{x}_{u 1}$.

Note that (9) coincides with the linear controller $u=$ $-K x$ near the origin and hence asymptotic (exponential) stability is automatically ensured in a region around the origin if $A-B K$ is Hurwitz. The following sections demonstrate that, under mild assumptions, (9) actually achieves asymptotic stability with a region of attraction equal to the null controllable region.

\section{Analysis Elements}

To prove stability for the systems presented, we will define a group of regions and show that these regions are positively invariant for the closed-loop system trajectories. We will further show that the trajectories starting in any of these regions reach a smaller region contained in the originating region and ultimately converge to the origin of the state-space. In this section we present the required definitions and assumptions that provide the construction elements for the subsequent results.

\subsection{Critical Points}

The closed-loop system (2)-(3), (9) is characterised by a piecewise affine controller. Piecewise affine controllers divide a system's controllable region into subregions each with a different control law. Hence, within each subregion the behaviour of the system is dominated by the location of a pseudo-equilibrium point (that is an equilibrium that would arise should the domain of a subregion be extended to include the whole state space) resulting from the application of the subregion's control law.

For the closed-loop system (2)-(3), (9) there are three subregions, which are governed by one linear and two saturated controllers. The linear control region $\underline{u} \leq-K x \leq$ $\bar{u}$ has a stable equilibrium point at the origin, and the saturated control regions corresponding to $u=\bar{u}$ and $u=\underline{u}$ have the pseudo-equilibrium points defined below. For example the equilibrium point for $u=\bar{u}$ is on the open boundary of the null controllable set (in the unstable state direction) and inside the stable subspace of the $u=\underline{u}$ controller.

Definition 2 (Pseudo-equilibrium Points) These points are denoted by $x^{e+}$ and $x^{e-}$ and are defined by, $A x^{e+}+B \underline{u}=0$ and $A x^{e-}+B \bar{u}=0$. Using (3), we obtain,

$$
\begin{aligned}
& x^{e+} \triangleq\left[\begin{array}{ll}
x_{u 1}^{e+} & x_{s}^{e+T}
\end{array}\right]^{T}=\left[\begin{array}{llll}
x_{u 1}^{e+} & x_{s 1}^{e+} & \cdots & x_{s q}^{e+}
\end{array}\right]^{T} \\
& x^{e-} \triangleq\left[\begin{array}{llll}
x_{u 1}^{e-} & x_{s}^{e-T}
\end{array}\right]^{T}=\left[\begin{array}{llll}
x_{u 1}^{e-} & x_{s 1}^{e-} & \cdots & x_{s q}^{e-}
\end{array}\right]^{T}
\end{aligned}
$$

where $x_{u 1}^{e+}=-b_{u 1} \underline{u} / a_{u 1}, x_{u 1}^{e-}=-b_{u 1} \bar{u} / a_{u 1}, x_{s i}^{e+}=$ $-b_{s i} \underline{u} / a_{s i}$ and $x_{s i}^{e-}=-b_{s i} \bar{u} / a_{s i}$ for $i=1, \ldots, q$.

We will also require the following $\varepsilon$-perturbation of the pseudo-equilibrium points (16) and (17).

Definition 3 ( $\varepsilon$ Pseudo-equilibrium Points) These points are denoted by $\underline{x}$ and $\bar{x}$ and for a given $\varepsilon=\left(\varepsilon_{u 1}, \varepsilon_{s}\right)=\left(\varepsilon_{u 1}, \varepsilon_{s 1}, \ldots, \varepsilon_{s q}\right)>0$ they are defined as follows:

$$
\begin{aligned}
& \underline{x} \triangleq\left[\begin{array}{ll}
\underline{x}_{u 1} & \underline{x}_{s}^{T}
\end{array}\right]^{T}=\left[\begin{array}{llll}
\underline{x}_{u 1} & \underline{x}_{s 1} & \cdots & \underline{x}_{s q}
\end{array}\right]^{T}, \\
& \bar{x} \triangleq\left[\begin{array}{llll}
\bar{x}_{u 1} & \bar{x}_{s}^{T}
\end{array}\right]^{T}=\left[\begin{array}{llll}
\bar{x}_{u 1} & \bar{x}_{s 1} & \cdots & \bar{x}_{s q}
\end{array}\right]^{T},
\end{aligned}
$$

where $\underline{x}_{u 1}=x_{u 1}^{e-}+\varepsilon_{u 1}, \bar{x}_{u 1}=x_{u 1}^{e+}-\varepsilon_{u 1}, \underline{x}_{s i}=x_{s i}^{e+}-\varepsilon_{s i}$ and $\bar{x}_{s i}=x_{s i}^{e-}+\varepsilon_{s i}$ for $i=1, \ldots, q$.

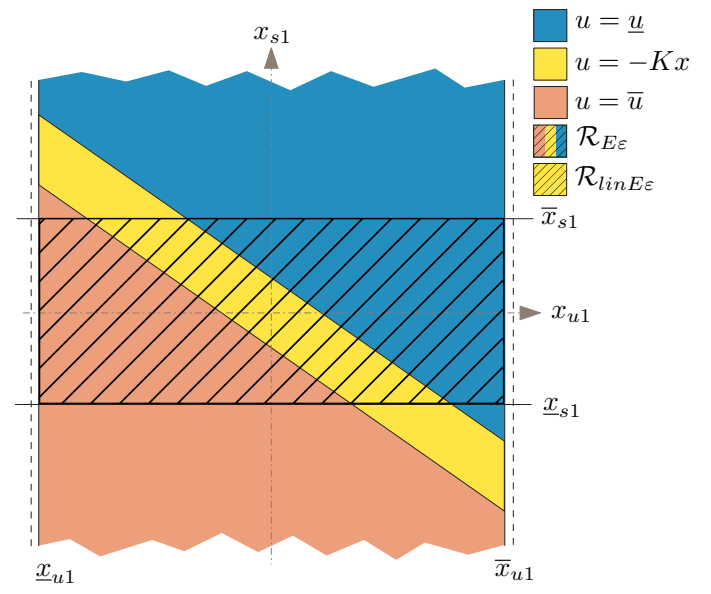

Fig. 2. Analysis planes and regions in $\mathbb{R}^{2}$.

\subsection{Analysis Regions}

Our first analysis region is an axis-aligned polytope that is delimited by hyperplanes passing through the $\varepsilon$ pseudo-equilibrium points given in Definition 3. These hyperplanes are illustrated for the case of $\mathbb{R}^{2}$ in Fig. 2.

Definition 4 (Pseudo-equilibrium $\varepsilon$ Region) This region, denoted by $\mathcal{R}_{E \varepsilon}$, is the defined as

$$
\mathcal{R}_{E \varepsilon} \triangleq\left\{x \in \mathbb{R}^{n}: \begin{array}{c}
\underline{x}_{u 1} \leq x_{u 1} \leq \bar{x}_{u 1} \quad \text { and } \\
\underline{x}_{s} \leq x_{s} \leq \bar{x}_{s}
\end{array}\right\}
$$

where $\underline{x}_{u 1}, \bar{x}_{u 1}, \underline{x}_{s}$ and $\bar{x}_{s}$, are as in (18) and (19).

Our next analysis region represents the subset of the $\mathcal{R}_{E \varepsilon}$ polytope where the control is linear $(-K x)$.

Definition 5 (Linear Pseudo-equilibrium $\varepsilon$ Region) This region denoted by $\mathcal{R}_{\text {linEs }}$, is defined as

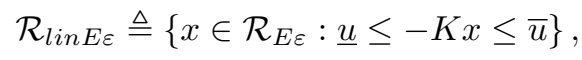


where $\mathcal{R}_{E \varepsilon}$ is as in Definition 4.

As an illustration of (20) and (21), Fig. 2 shows $\mathcal{R}_{\max -\varepsilon}$ (cf. Definition 1) and the analysis regions for $\mathbb{R}^{2}$.

\subsection{Controller Gain $K$ and Parameter $\varepsilon$}

To facilitate the results presented in the sequel we require the controller gain $K$ and parameter $\varepsilon$ (see Definition 3 ) in (9) to satisfy the following assumption.

Assumption 1 (Controller Parameters) The gain $K=\left[\begin{array}{llll}k_{u 1} & k_{s 1} & \cdots & k_{s q}\end{array}\right]$ in (9) is such that $A-B K$ is Hurwitz and the following two inequalities hold.

$$
\begin{aligned}
& \sum_{i \in \mathcal{I}^{+}} k_{s i} b_{s i} \frac{a_{u 1}}{a_{s i}} \bar{u}+\sum_{i \in \mathcal{I}^{-}} k_{s i} b_{s i}\left(\frac{a_{u 1}}{a_{s i}} \underline{u}+\bar{u}-\underline{u}\right)+ \\
& \left(k_{u 1} b_{u 1}-a_{u 1}\right) \bar{u} \geq \alpha, \\
& \sum_{i \in \mathcal{I}^{+}} k_{s i} b_{s i} \frac{a_{u 1}}{a_{s i}} \underline{u}+\sum_{i \in \mathcal{I}^{-}} k_{s i} b_{s i}\left(\frac{a_{u 1}}{a_{s i}} \bar{u}+\underline{u}-\bar{u}\right)+ \\
& \left(k_{u 1} b_{u 1}-a_{u 1}\right) \underline{u} \leq-\alpha,
\end{aligned}
$$

for some $\alpha>0$, where

$$
\begin{aligned}
& \mathcal{I}^{+} \triangleq\left\{i \in\{1,2, \ldots, q\}: k_{s i}>0\right\} \\
& \mathcal{I}^{-} \triangleq\left\{i \in\{1,2, \ldots, q\}: k_{s i}<0\right\}
\end{aligned}
$$

In addition, let $\varepsilon=\left(\varepsilon_{u 1}, \varepsilon_{s 1}, \ldots, \varepsilon_{s q}\right)>0$ satisfy

$$
\varepsilon_{u 1} \leq-\sum_{i=1}^{q} \frac{\left|k_{s i}\right| a_{s i}}{k_{u 1} a_{u 1}} \varepsilon_{s i} \text { and } \sum_{i=1}^{q}\left|k_{s i}\right| \varepsilon_{s i}\left(a_{u 1}-a_{s i}\right)<\alpha
$$

This places restrictions on the admissible controller gains ${ }^{5} K$ and parameter $\varepsilon$ to ensure that certain geometric properties hold for region $\mathcal{R}_{\text {linE }}$ defined in (21).

\section{Properties of the Analysis Regions}

In this section we show that the regions defined in Section 4.2 are nested and that each is positively invariant for the closed-loop system trajectories and attractive of trajectories originating in a containing region.

Lemma 1 (Nesting of Analysis Regions) The re-

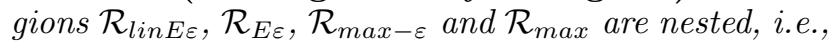

$$
\mathcal{R}_{\text {linE } \varepsilon} \subset \mathcal{R}_{E \varepsilon} \subset \mathcal{R}_{\max -\varepsilon} \subset \mathcal{R}_{\max }
$$

\footnotetext{
${ }^{5}$ Selecting $K$ to be of the form $K=\left[\begin{array}{llll}k_{u 1} & 0 & \ldots & 0\end{array}\right]$ results in one possible set of gains satisfying Assumption 1 (see $[10]$ Remark 3.3 for the proof).
}

PROOF. Immediate from (4) and Definitions 5, 4, 1.

Lemma 2 (Invariance of Analysis Regions) For the closed-loop system (2)-(3), (9), and under Assumption 1 the following statements are true.

(1) $\mathcal{R}_{\max -\varepsilon}$ defined in (5) is positively invariant.

(2) $\mathcal{R}_{E \varepsilon}$ defined in (20) is positively invariant.

(3) $\mathcal{R}_{\text {linE }}$ defined in (21) is positively invariant. Moreover, the origin is asymptotically stable with a region of attraction that includes $\mathcal{R}_{\text {linE }}$.

PROOF. See [10], (1) Lemma 3.6, (2) Lemma 3.8 and (3) Lemma 3.10.

Lemma 3 (Attractiveness of Analysis Regions) For the closed-loop system (2)-(3), (9), the following statements are true.

(1) All states in the region $\mathcal{R}_{\max }$ (see (4)), enter $\mathcal{R}_{\max -\varepsilon}($ see (5)) in finite time.

(2) All states inside $\mathcal{R}_{\max -\varepsilon}\left(\right.$ see (5)) but outside $\mathcal{R}_{E \varepsilon}$ (see (20)) enter $\mathcal{R}_{E \varepsilon}$ in finite time.

(3) All states inside $\mathcal{R}_{E \varepsilon}$ (see (20)) but outside the inte-

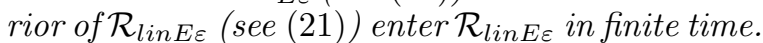

PROOF. See [10], (1) Lemma 3.7, (2) Lemma 3.9 and (3) Lemma 3.11.

\section{Maximal Controllability}

In this section we present the main result of this paper, which shows that the controller (9) asymptotically stabilises system (2)-(3), achieving a region of attraction that is arbitrarily close to the null controllable region.

Theorem 1 (Maximal Controllability) For the closed-loop system (2)-(3), (9) and a selection of $K$ and $\varepsilon$ that satisfy Assumption 1, the origin is asymptotically stable with a region of attraction $\mathcal{R}_{\max }$.

PROOF. The proof of stability consists in showing that for the closed-loop system (2)-(3), (9), the trajectories starting in $\mathcal{R}_{\max }, \mathcal{R}_{\max -\varepsilon}$ and $\mathcal{R}_{E \varepsilon}$ converge to $\mathcal{R}_{\text {linE } \varepsilon}$ in finite time. Then, inside $\mathcal{R}_{\text {linE\& }}$, the trajectories asymptotically converge to the origin.

First consider points in the region $\left\{x: x \in \mathcal{R}_{\max }\right.$ and $x \notin$ int $\left.\mathcal{R}_{\max -\varepsilon}\right\}$. By Lemma 3 (1), all trajectories starting in this region reach $\mathcal{R}_{\max -\varepsilon}$ in finite time.

Next consider points in the region $\left\{x: x \in \mathcal{R}_{\max -\varepsilon}\right.$ and $x \notin$ int $\left.\mathcal{R}_{E \varepsilon}\right\}$. By Lemma 3 (2) all trajectories starting in this region must reach region $\mathcal{R}_{E \varepsilon}$ in finite time. 
Finally, consider points in the region $\{x: x \in$

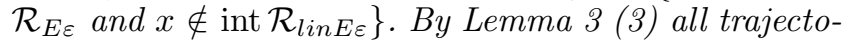
ries starting in this region enter $\mathcal{R}_{\text {linE }}$ in finite time.

By Lemma 2 (3), $\mathcal{R}_{\text {linEs }}$ is positively invariant and the origin is asymptotically stable with a region of attraction that includes $\mathcal{R}_{\text {linE } \varepsilon}$. Therefore, all trajectories starting in $\mathcal{R}_{\max }$ converge asymptotically to the origin, proving the result.

\section{$7 \quad$ Example}

To illustrate the results presented above, we apply the controller presented in Section 3 to a system of the form (2)-(3) with $a_{u 1}=1, A_{s}=-1$ and $b_{u 1}=B_{s}=1$, with input constraints $-1 \leq u \leq 1$. From (4), the null controllable region is $-1<x_{u 1}<1$ and by selecting $\varepsilon_{u 1}=0.01$ the implicit constraints are $-0.99 \leq x_{u 1} \leq$ 0.99 . The gain $K$ in (9) is taken as $K=[2.40 . \overline{4}]$, which satisfies Assumption 1.

Fig. 3 represents a small subregion of the closed-loop system's null controllable set, whose boundaries are identified by the two dashed lines located at $x_{u 1}=-1$ and $x_{u 1}=1$. A number of initial states were selected and their state evolution trajectories plotted. Each initial state is depicted by a circle and its evolution trajectory is depicted by a solid line. Further, the shaded region in the centre of the figure represents the unsaturated or linear control region of the closed-loop system. From the figure we see that all selected initial states have trajectories that converge to the origin. Further, we note that a portion of some trajectories exhibit sliding along the implicit constraint boundaries, thus confirming the trajectory behaviour predicted by the analysis in the previous sections. The simulations also suggest that the closedloop system exhibits maximal controllability.

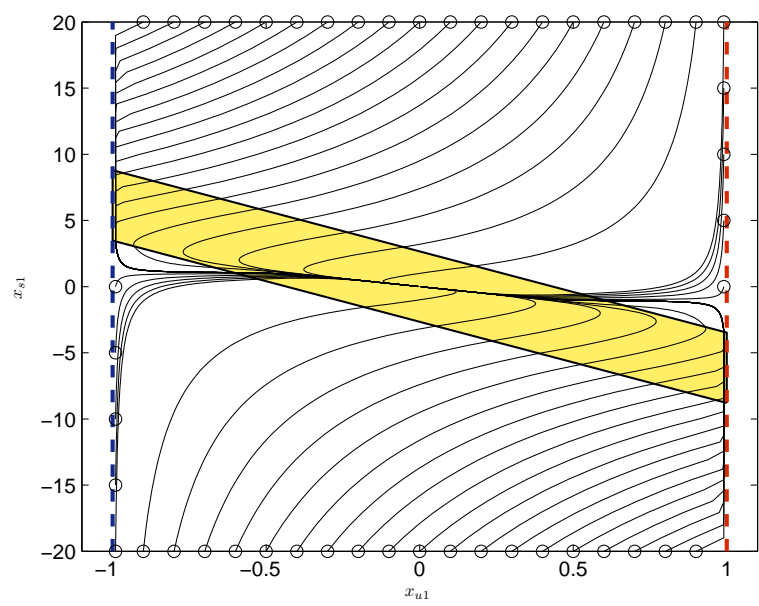

Fig. 3. State trajectories for an array of initial states.

\section{Extensions}

This section briefly describes some extensions to this maximal controllability approach and its limitations with respect to a broader class of systems. In all cases the presented analysis relies on the resulting null controllable set of the system and whether its boundaries are sufficiently "simple" to be easily applied as an implicit constraint. For the case where the resulting systems have null controllable sets that are not sufficiently simple the authors have developed a different technique known as RPMPC [10].

Two possible extensions are the introduction of multiple inputs and multiple unstable modes. Three distinct types of systems result from the combination of these two extensions and are discussed in the context of the described maximal controllability approach.

\subsection{One Unstable Mode with Multiple Inputs}

Adding multiple inputs to systems with a single unstable mode is such that the resulting null controllable set is of the same shape as the one for a single input with the redefinition of $\underline{u}$ and $\bar{u}$, and the implicit constraints as

$$
\begin{aligned}
& \underline{u}=\left[\begin{array}{lll}
\underline{u}_{1}^{s a t} & \ldots & \underline{u}_{m}^{s a t}
\end{array}\right]=\underset{u_{1}, \ldots, u_{m} \in \mathbb{U}}{\arg \min }-\sum_{i=1}^{m} b_{u i} / a_{u 1} u_{i} \\
& \bar{u}=\left[\begin{array}{lll}
\bar{u}_{1}^{s a t} & \ldots & \bar{u}_{m}^{s a t}
\end{array}\right]=\underset{\substack{u_{1}, \ldots, u_{m} \in \mathbb{U} \\
\arg \max }}{\arg } b_{i=1}^{m} / a_{u 1} u_{i}
\end{aligned}
$$

and

$$
\underline{x}_{u 1}=-\sum_{i=1}^{m} b_{u i} / a_{u 1} \underline{u}_{i}^{s a t} \text { and } \bar{x}_{u 1}=-\sum_{i=1}^{m} b_{u i} / a_{u 1} \bar{u}_{i}^{s a t}
$$

Hence, under similar assumptions such systems can result in maximal controllability.

\subsection{Multiple Unstable Modes and Multiple Inputs}

For systems with $p>1$ unstable modes and $m>1$ inputs, the null controllable sets become more complicated and may be maximally controlled by the above described technique if additional conditions are met. Applicable systems are those where the number of inputs $m \geq p$ and where each column of $B_{u}$, the input weightings of the unstable subsystem, contains at least one non-zero entry. If these conditions are met, the resulting null controllable set is an axis aligned $p$-orthotope, in $\mathbb{R}^{p}$, for the unstable states and unbounded for the stable modes. Similarly, $\underline{u}_{i}$ and $\bar{u}_{i}$, and the implicit constraints for $i=1, \ldots, p$ are 
redefined as follows.

$$
\begin{aligned}
& \underline{u}_{i}=\left[\begin{array}{lll}
\underline{u}_{i 1}^{s a t} & \cdots & \underline{u}_{i m}^{s a t}
\end{array}\right]=\underset{u_{1}, \ldots, u_{m} \in \mathbb{U}}{\arg \min }-\sum_{j=1}^{m} b_{u i j} / a_{u i} u_{j} \\
& \bar{u}_{i}=\left[\begin{array}{lll}
\bar{u}_{i 1}^{s a t} & \ldots & \bar{u}_{i m}^{s a t}
\end{array}\right]=\underset{u_{1}, \ldots, u_{m} \in \mathbb{U}}{\arg \max }-\sum_{j=1}^{m} b_{u i j} / a_{u i} u_{j}
\end{aligned}
$$

and

$$
\underline{x}_{u i}=-\sum_{j=1}^{m} b_{u i j} / a_{u i} \underline{u}_{i j}^{s a t} \text { and } \bar{x}_{u i}=-\sum_{j=1}^{m} b_{u i j} / a_{u i} \bar{u}_{i j}^{s a t}
$$

If these conditions are not met the resulting null controllable set does not have a "simple" closed form representation of its boundaries which means that our given technique no longer applies.

\subsection{Multiple Unstable Modes and a Single Input}

For this class of systems the null controllable set does not have a "simple" closed form description of its boundary and as such does not satisfy the precondition of the presented technique.

\section{Conclusions and Discussions}

In this paper we have observed that the combination of applied constraints and system dynamics can result in "implicit constraints", which describe the boundaries of the null controllable set of a system. If the characterisation of the null controllable set is sufficiently simple, these implicit constraints can be easily incorporated in the formulation of the control problem. We have applied this technique to a control strategy and shown that for a non-trivial class of systems, the resulting closed-loop system has a region of attraction equal to the null controllable set.

Although the analysis presented was in terms of a continuous-time controller, the successful application of MPC with implicit constraints to the magnetic suspension system described in Section 1.1 suggests that similar results would apply to MPC. Thus, the proposed technique may provide a very simple addition to an MPC formulation (i.e., the addition of the implicit state constraints) which would allow the system to be stabilised, by MPC, over the entire controllable space. By comparison stabilisation of MPC using terminal set constraints makes the controllable set horizon length dependent and therefore, due to computational complexity, very conservative with respect to the null controllable set of the system.

Note that our results suggest that it would suffice to consider horizon 1 to achieve maximal controllability by means of the introduction of implicit constraints. On the other hand, with a horizon length of 1 and initial states with large stable state values, the saturated control regions produce trajectories that reach the implicit boundary and slide along the boundary. When the horizon length is increased more control regions are generated hence a smaller subset of initial states will result in the trajectories reaching the implicit boundary. As a result shorter settling times may be possible in some cases and an overall lower control cost may result.

\section{References}

[1] F. Blanchini and S. Miani. Set-theoretic Methods in Control. Springer, Dordrecht, July 2005. ISBN: 9780817646066.

[2] E.F. Camacho. Constrained generalized predictive control. IEEE T. Automat. Contr., 38(2):327-332, 1993.

[3] R.A. DeCarlo, S.H. Zak, and S.V. Drakunov. Variable structure, sliding-mode controller design. In William S. Levine, editor, The Control Handbook, pages 941-951. CRC Press, 1996.

[4] J-Y. Favez, Ph. Mullhaupt, B. Srinivasan, D. Bovin, and J.B. Lister. Enhancing tokamak control given power supply voltage saturation. ICALEPCS2003, pages 40-42, 2003.

[5] A.F. Filippov. Differential equations with discontinuous right hand sides. Translations of the American Mathematical Society, 62, 1960.

[6] G.C. Goodwin, M.M. Seron, and J.A. De Doná. Constrained Control and Estimation: An Optimization Approach. Springer, 2004.

[7] T. Hu, Z. Lin, and L. Qiu. Stabilization of exponentially unstable linear systems with saturating actuators. IEEE T. Automat. Contr., 46(6):973-979, Jun 2001.

[8] H. Khalil. Nonlinear Systems. Prentice-Hall, 3rd. edition, 2002 .

[9] D.Q. Mayne, J.B. Rawlings, C.V. Rao, and P.O.M. Scokaert. Constrained model predictive control: Stability and optimality. Automatica, 36:789-814, 2000.

[10] Adrian Medioli. Maximal Controllability via Reduced Complexity Model Predictive Control. PhD thesis, University of Newcastle. FEBE, SEECS, 2008. http://hdl.handle.net/1959.13/30688.

[11] S. Sastry. Nonlinear Systems. Analysis, Stability and Control. Springer, 1999.

[12] E. Schuster, M.L. Walker, D.A. Humphreys, and M. Krstic. Plasma vertical stabilization in the presence of coil voltage saturation in the diii-d tokamak. In Proceedings of the American Control Conference, Denver, Colorado, 2003.

[13] L. Scibile and B. Kouvaritakis. Stability region for a class of open-loop unstable linear systems: theory and application. Automatica, 36:37-44, 2000.

[14] A.R. Teel. Anti-windup for exponentially unstable linear systems. Int. J. Robust Nonlin., 9(10):701-716, 1999. 